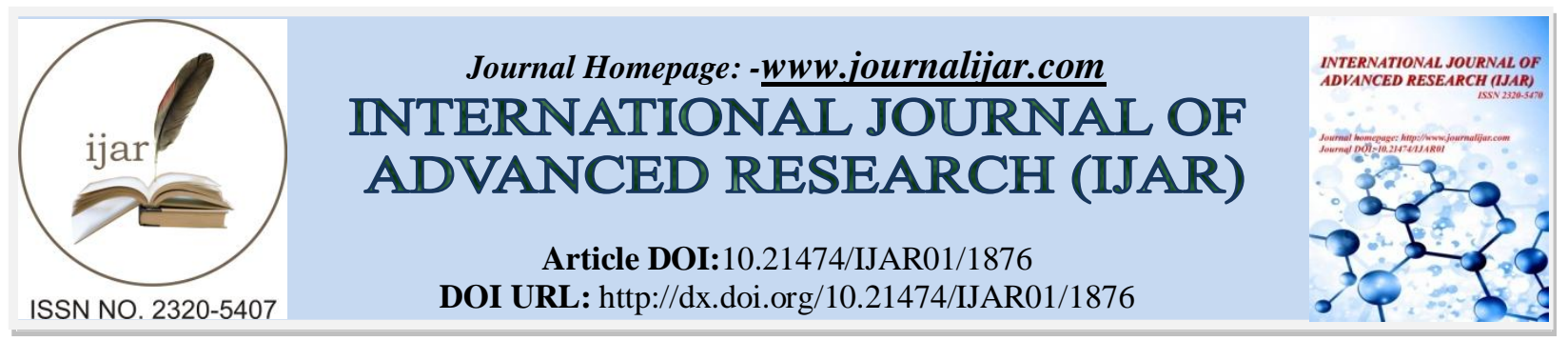

RESEARCH ARTICLE

\title{
THE ORDERING AMBIGUITY AND POSITION DEPENDENT MASS FOR NON- RELATIVISTIC QUANTUM PARTICLE WITH INTER-DIMENSIONAL (D=1)
}

Ashwaq Eyad Alaakol.

Muthanna University, College of science, Physics Department, Al- Muthanna, Samawa, Iraq.

\section{Manuscript Info}

Manuscript History

Received: 11 August 2016

Final Accepted: 22 September 2016

Published: October 2016

Key words:-

Position dependent mass, PCT Method,

Von -Roos ordering, Eigenfunction

\section{Abstract}

By revisiting free particle $v(x)=0$ endowed with position dependent mass $m(x)=\frac{1}{\left(1+(\kappa x)^{2}\right)^{2}}$ has $d=1$ (the inter-dimensional). Starting with the kinetic energy operator, we will try to solve the equation by simplification mathematical method to find energy spectrum, then get stander form for the energy whose dependent on the ordering ambiguity.

Copy Right, IJAR, 2016,. All rights reserved.

\section{Introduction:-}

Position dependent mass quantum particles in non-relativistic quantum theory have inspired researchers in the last few years [1-4]. They found new useful and interesting models help them in physical obstructions [5], semiconductors [6], quantum dot [7], quantum liquids [8], He-clusters [9] and metal clusters [10].

Quantum mechanical particle endowed with PDM erupt in the system of quantum mechanics to solve more problems of a delicate nature like example: the $m(x)$, kinetic energy and the momentum operator does not commute [11].

In other side, they try to find a solution for the Schrödinger equation in d- dimensional by dependent a canonical transformation (PCT) method [12,13 ] under task change variables [14-16]. The eigenvalues and eigenfunctions are mapped for the exact solutions for Schrödinger equation [17-19].

Recently, PCT for PDM-quantum particle introduced in d- dimensional [1]. The inter-one degeneracies associated with the isomorphism between angular momentum $l$ and dimensionality $d$ builds up the ladder of excited states for any given values e.g. nonzero land the radial quantum number $n_{r}$. By choosing the parameters $\alpha=\gamma a n d \beta=$ -1with the PCT method, it lead to get the Schrödinger equation with the effective potential( Poschl-Teller potential) [20].

Here, we choose a free particle with $v(x)=0$, the inter - dimensional $d=1$ and keeping on the von- Roos ordering in the general form $\alpha+\beta+\gamma=-1$, we will try to find a new formula for energy spectrum depend on position dependent mass parameters.

Time -independent Schrödinger equation in one- dimensional by PCT method:-

The stander form time-independent Schrödinger equation is given:

$-\frac{\hbar^{2}}{2 m} \frac{\partial^{2}}{\partial x^{2}} \psi(x)+v(x) \psi(x)=E \psi(x)$ 
In which $\mathrm{v}(\mathrm{x})$ is a real potential and $\mathrm{E}$ is the energy of the particle.

Further, kinetic energy of quantum particle with position-dependent mass in one dimension is given by [1]

$T=\frac{1}{4}\left(m^{\alpha} p m^{\beta} p m^{\gamma}+m^{\gamma} p m^{\beta} p m^{\alpha}\right)$

In which $\alpha+\beta+\gamma=-1$ with $p=-i \hbar \frac{d}{d x}$ and $m=m(x)$.

Using equation (1) the time-independent Schrödinger equation for a position -dependent mass particle reads:

$\frac{1}{4}\left(m^{\alpha} p m^{\beta} p m^{\gamma}+m^{\gamma} p m^{\beta} p m^{\alpha}\right) \psi(x)+v(x) \psi(x)=E \psi(x)$

Bysimplifying Eq.(3) one can obtain:

$\frac{-\hbar^{2}}{4}\left[\frac{2}{m} \psi^{\prime \prime}+(-2) \frac{m^{\prime}}{m^{2}} \psi^{\prime}+\frac{m^{\prime 2}}{m^{3}}(\beta(\gamma+\alpha)+\gamma(\gamma-1)+\alpha(\alpha-1)) \psi+\frac{(-1-\beta)}{m^{2}} m^{\prime \prime} \psi\right]+v \psi=E \psi$

Where a prime mean derivative with respect to its argument.

Using point canonical transformation (PCT) by introducing $\psi(x)=g(x) \varphi(q(x))$ and the latter equation reads:

$$
\frac{-\hbar^{2}}{4}\left[\begin{array}{c}
\frac{2}{m}\left(g^{\prime \prime}(x) \varphi(q(x))+2 g^{\prime}(x) q^{\prime} \varphi^{\prime}(q(x))+g(x) q^{2} \varphi^{\prime \prime}(q(x))\right)+(-2) \frac{m^{\prime}}{m^{2}} \\
\left(g^{\prime}(x) \varphi(q(x))+g(x) q^{\prime} \varphi^{\prime}(q(x))\right)+\frac{m^{2}}{m^{3}}(\beta(\gamma+\alpha)+\gamma(\gamma-1)+\alpha(\alpha-1)) g(x) \varphi(q(x)) \\
+\frac{(-1-\beta)}{m^{2}} m^{\prime \prime} g(x) \varphi(q(x)) \\
+V(q(x)) g(x) \varphi(q(x))=E g(x) \varphi(q(x))
\end{array}\right]
$$

To have a Schrödinger equation, we set $\varphi^{\prime}(q(x))$ to be zero, therefore $g^{2}(x) q^{\prime}(x)=m(x)$.

After that, we set the coefficient of $\varphi^{\prime}(q(x))$ to be constant, so $q^{2}(x)=m(x)$.

$$
\frac{-\hbar^{2}}{4}\left[\begin{array}{c}
\frac{-3}{16} m(x)^{\frac{-7}{4}} m^{2}+\left(\frac{1}{4}\right) m(x)^{\frac{-3}{4}} m^{\prime \prime} \varphi(q(x))+m(x)^{\frac{1}{4}} q^{\prime 2} \varphi^{\prime}(q(x))+(-2) \frac{m^{\prime}}{m^{2}}\left(\frac{1}{4}\right) m(x)^{\frac{-3}{4}} m^{\prime} \\
\varphi(q(x))+\frac{m^{2}}{m^{3}}\left(m(x)^{\frac{1}{4}}\right)(\beta(\gamma+\alpha)+\gamma(\gamma-1)+\alpha(\alpha-1)) \varphi(q(x))+ \\
\frac{(-1-\beta)}{m^{2}} m^{\prime \prime} m(x)^{\frac{1}{4}} \varphi(q(x)) \\
+V(q(x)) m(x)^{\frac{1}{4}} \varphi(q(x))=E m(x)^{\frac{1}{4}} \varphi(q(x))
\end{array}\right]
$$

Dividing Eq.(6) by $m(x)^{\frac{1}{4}}$ yields:

$$
\frac{-\hbar^{2}}{4}\left[\begin{array}{c}
\frac{2}{m}\left(\left(\frac{-3}{16} \frac{m^{2}}{m(x)^{2}}+\frac{1}{4} \frac{m^{\prime \prime}}{m(x)}\right) \varphi(q(x))+m(x) \varphi^{\prime \prime}(q(x))\right)-\frac{1}{2} \frac{m^{2}}{m^{3}}\left(\frac{1}{4}\right) \\
\varphi(q(x))+\frac{m^{2}}{m^{3}}(\beta(\gamma+\alpha)+\gamma(\gamma-1)+\alpha(\alpha-1)) \varphi(q(x))+ \\
(-1-\beta) \frac{m^{\prime \prime}}{m^{2}} \varphi(q(x)) \\
=E \varphi(q(x))
\end{array}\right]+V(q(x)) \varphi(q(x))
$$

Hence, we obtain Schrödinger equation that reads:

$\left(-\frac{1}{2} \frac{d^{2}}{d q^{2}}+V_{e f f}\right) \varphi(q(x))=\frac{E}{\hbar^{2}} \varphi(q(x))$

With the effective potential for free particle $V(q(x))=0$ : 
$V_{e f f}=\frac{7}{32} \frac{m^{2}}{m^{3}}-\left(\frac{1}{8}-\frac{(-1-\beta)}{4}\right) \frac{m^{\prime \prime}}{m^{2}}-\frac{1}{4} \frac{m^{2}}{m^{3}}(\beta(\gamma+\alpha)+\gamma(\gamma-1)+\alpha(\alpha-1))$

Introducing example of vanishing mass withSchrödinger equation:-

The form of mass that is used here:

$m(x)=\frac{1}{\left(1+(\kappa x)^{2}\right)^{2}} \quad ; k:$ isaconstant

Which in turn gets:

$q(x)=\int_{0}^{x} \frac{d x}{\left(1+(\kappa x)^{2}\right)^{2}} \Rightarrow q(x)=\frac{1}{k} \tan ^{-1}(k x)$

Also one finds, from the latter equation:

$\tan (k q(x))=k x$

By considering these results into the equation (10), one finds:

$m(x)=\cos ^{4}(k q)$

$m^{\prime}(x)=-4 k \cos ^{3}(k q) \sin (k q) q^{\prime}(x)$

$m^{\prime \prime}(x)=-4 k\left(-3 k \cos ^{2}(k q) \sin ^{2}(k q) q^{2}+k \cos ^{4}(k q) q^{2}+\cos ^{3}(k q) \sin (k q) q^{\prime \prime}\right)$

Then the effective potential will give as:

$\left.\left.V_{e f f}=k^{2} \tan ^{2}(k q)+\frac{k^{2}}{2}-(-1-\beta)\left(k^{2}\left(5 \tan ^{2}(k q)-1\right)\right)-4\left(\begin{array}{c}\beta(\gamma+\alpha)+ \\ \gamma(\gamma-1)+\alpha(\alpha-1)\end{array}\right)\right) \tan ^{2}(k q)\right)$

$V_{e f f}=-\frac{k^{2}}{2}-k^{2} \beta+\left(\lambda(\lambda-1) k^{2} \tan ^{2}(k q)(17)\right.$

Where $\left(\lambda(\lambda-1)=6 k^{2}+k^{2} \beta-4(\beta(\gamma+\alpha)+\gamma(\gamma-1)+\alpha(\alpha-1))\right.$

So Schrödinger equation will be as follows:

$\left(-\frac{1}{2} \frac{d^{2}}{d q^{2}}+\frac{\left(\lambda(\lambda-1) k^{2}\right.}{\cos ^{2}(k q)}\right) \varphi(q(x))=\epsilon \varphi(q(x))$

here $\epsilon=\frac{E}{\hbar^{2}}+\frac{k^{2}}{\hbar^{2}}+k^{2} \beta$.

Now we will solve the equation (18) by change the variable, by considering a new function which is $\varphi(z)=$ $\cos ^{\lambda}(k q) F(z)$ where $z=\sin ^{2}(k q)$ that is lead to $(z)=(1-\mathrm{z})^{\lambda}(k q) F(z)$.

Hence we find our equation in hypergeometric form:

$z(1-z) F^{\prime \prime}+\frac{1}{4}(2-4(1+\lambda) z) F^{\prime}+\frac{2 \varepsilon-\lambda^{2} k^{2}}{4 k^{2}} F=0$

By comparing Eq. (19) with the solution of the second order hypergeometric equation - which in the form ( $x(1-$ $\left.x) F^{\prime \prime}+(c-(a+b+1) x) F^{\prime}+a b F=0\right)-$ that it yields: 


$$
b \circ=\frac{\lambda \pm \sqrt{\lambda^{2}-4\left(\frac{\lambda^{2}}{4}-\frac{\varepsilon}{2 k^{2}}\right)}}{2}
$$

So that :

$a=\frac{\lambda-\sqrt{\frac{2 \epsilon}{k^{2}}}}{2}$ and $b=\frac{\lambda+\sqrt{\frac{2 \epsilon}{k^{2}}}}{2}$

$\frac{1}{2}\left(\lambda-\sqrt{\frac{2 \varepsilon}{k^{2}}}\right)=-a \quad ;(a)$ is an integer number.

And $\varepsilon_{n}=2 k^{2}\left(a+\frac{\lambda}{2}\right)^{2}$,

Now trying to get the energy by using the solution ofthe second independent Hyprogeomtric equation which it is read :

$F(x)=c_{1} F(a, b, c ; x)+c_{2} x^{1-c} F(a+1-c, b+1-c, 2-c ; x)$

Using equation above, one can obtain:

$E_{n}=2 k^{2}\left(a+\frac{\lambda}{2}\right)^{2}-\frac{k^{2}}{2}-k^{2} \beta$

The last Eq.(21) represent to the total energy .

\section{Conclusion:-}

In this work we have considered the one dimensional quantum particle with vanishing position- dependent mass and $v(x)=0$. Our main concern here to introduce the general form of the Schrödinger equation with the ordering ambiguity in its stander form $\alpha+\beta+\gamma=-1$. In other word, the energy spectrum depends on the parameters of position dependent mass $\alpha, \beta a n d \gamma$. One can see the larger part of energy depend on the ordering ambiguity. Add to this the effect potential ( Poschl-Teller potential) here has a new option with the von- Roos ordering. The exact solution mapped to match the attendant setting of our free particle with the position-dependent mass $(m(x)=$ $\frac{1}{\left(1+(k x)^{2}\right)^{2}}$.

\section{References:-}

1. O. Mustafa, S.H Mazharimousavi,"Point canonical transformationd-dimensionalregularization",arXiv :math-ph , 1, 2006.

2. S.H Mazharimousavi," Revisiting the displacement operator for quantum systems with position-dependent mass",arxiv: quant-ph, 2,1203-2799, 2012.

3. R De, R Dutt and U Sukhatme, "Mapping of shape invariant potentials under point canonical transformations", J. Phys A / Math and Gen, 25, $843,1992$.

4. G Junker "Remarks on the local time rescaling in path integration", J. Phys. A/MathandGen, v23 L881, 1990.

5. A Puente and M Casas," Non-local energy density functional for atoms and metal clusters", Compute. Mater. Sci.,2, 441-449, 1994.

6. Bastard G " Wave Mechanics Applied to Semiconductor Heterostructures" ,LesEditions de Physique, Les Ulis, 1988.

7. L I Serra and E Lipparini ," Spin response of unpolarized quantum dots ", Euro.PhysLett., 40, 667-672, 1997.

8. F Arias de Saaverda, J Boronat, A Polls , and A Fabrocini, " Effective mass of one 4He atom in liquid 3He", Phys. Rev. B, 50, 4248 ,1994.

9. M Barranco , M Pi, S.M Gatica ., E.S Hemandez ., and J. Navarro," Structure and energetics of mixed 4He-3He drops", Phys.Rev. B, 56,8997 ,1997.

10. A Puente, L I Serra, and M Casas, " Dipole excitation of Na clusters with a non-local energy density functional", Z. Phys. D , 31, 283,1994.

11. A R Plastino, M Casas and A. Plastino," Bohmian quantum theory of motion for particles with positiondependent effective mass ", Phys. Lett. A, 281,297, 2001. 
12. C Quesne and V M Tkachuk, " Deformed algebras, position-dependent effectivemasses and curved spaces: an exactly solvable Coulomb problem", J. Phys. A / Math and Gen , $37,4267,2004$.

13. A D Alhaidari, " Solutions of the nonrelativistic wave equation with position-dependent effective mass", Phys. Rev. A, 66 042116, 2002.

14. J Liouville, " Sur le développement des fonctionsou parties de fonctions en series",J. Math. Pure Appl., 1 ,16$35,1837$.

15. M Znojil and G L'evai, "Comprehensive analysis of conditionally exactly solvable models", J. Math. Phys., 42, 1996-2007, 200.

16. M Znojil and G L'evai, "The Coulomb - harmonic oscillator correspondence in $\mathscr{P} \mathscr{T}$ symmetric quantum mechanics" Phys. Lett. A 271, 327-333,2000.

17. C Quesne, "First-order intertwining operators and position-dependent massSchrödinger equations in d dimensions" Ann. Phys. 321,1221- 1239, 2006.

18. S H Dong and M. Lozada-Cassou, "Exact solutions of the Schrödinger equation with the position-dependent mass for a hard-core potential" Phys. Lett. A, 337,313-320; 2005.

19. B Bagchi, A Banerjee, C Quesne and V M Tkachuk, "Deformed shape invariance and exactly solvable Hamiltonians with position-dependent effective mass" J. Phys. A;Math and Gen 38, 2929- 2945. 2005.

20. [O Mustafa and S.H Mazharimousavi, "Quantum particles trapped in a position-dependent mass barrier; a ddimensional recipe" /Phys.Lett.A, 358, 259- 261,2006. 